\title{
uPA/uPAR downregulation inhibits radiation-induced migration, invasion and angiogenesis in IOMM-Lee meningioma cells and decreases tumor growth in vivo
}

\author{
ODYSSEAS KARGIOTIS ${ }^{1 *}$, CHANDRAMU CHETTY ${ }^{1 *}$, VENKATESWARA GOGINENI ${ }^{1}$, \\ CHRISTOPHER S. GONDI ${ }^{1}$, SAI MURALIKRISHNA PULUKURI ${ }^{1}$, ATHANASSIOS P. KYRITSIS ${ }^{4,5}$, \\ MEENA GUJRATI ${ }^{2}$, JEFFREY D. KLOPFENSTEIN ${ }^{3}$, DZUNG H. DINH ${ }^{3}$ and JASTI S. RAO ${ }^{1,3}$ \\ Departments of ${ }^{1}$ Cancer Biology and Pharmacology, ${ }^{2}$ Pathology, and ${ }^{3}$ Neurosurgery, University of Illinois College \\ of Medicine at Peoria, Peoria, IL, USA; ${ }^{4}$ Neurosurgical Institute, ${ }^{5}$ Department of Neurology, \\ University of Ioannina Medical School, Ioannina, Greece
}

Received June 19, 2008; Accepted August 2, 2008

DOI: 10.3892/ijo_00000081

\begin{abstract}
Meningioma is a well-known tumor of the central nervous system, and is treated by surgical resection and/or radiation. Recently, ionizing radiation has been shown to enhance invasiveness of surviving tumor cells, and several proteolytic enzyme molecules, including urokinase plasminogen activator ( $\mathrm{UPA}$ ), seem to be upregulated after radiation. uPA and its receptor (UPAR) have been strongly implicated in tumor invasion, angiogenesis and progression. Hence, the tumor-associated uPA-uPAR system is considered a potential target for cancer therapy. In the present study, we show that radiation increases uPA levels in the IOMM-Lee meningioma cells, and subsequently, increases tumor invasion, migration and angiogenesis in vitro. Studies with signaling molecule inhibitors AG1478, U0126 and SB203580 (specific inhibitors of EGFR, MEK1/2 and p38 respectively) showed inhibition of uPA levels in both basal and irradiated-IOMM-Lee cells. The PI3K inhibitor (LY294002) and the AKT inhibitor (AKT inhibitor IV) also partially decreased uPA expression, whereas SP600125, a JNK inhibitor, did not affect uPA levels in either radiated or non-radiated cells. Further, a bicistronic plasmid construct with small interfering RNA (siRNA) against uPA and its receptor inhibited tumor invasion, migration and angiogenesis in radiation-treated IOMM-Lee cells. In addition, siRNA against uPA and its receptor inhibited subcutaneous
\end{abstract}

Correspondence to: Dr Jasti S. Rao, Department of Cancer Biology and Pharmacology, University of Illinois College of Medicine, One Illini Drive, Peoria, IL 61605, USA

E-mail: jsrao@uic.edu

${ }^{*}$ Contributed equally

Key words: uPA, uPAR, meningioma cells tumor growth in athymic nude mice in combination with radiation in a synergistic manner. Thus, the specific targeting of proteases via RNA interference could augment the therapeutic effect of radiation and prevent the adverse effects resulting from tumor cells that receive sublethal doses of radiation within the tumor mass.

\section{Introduction}

Meningiomas, which arise from the arachnoid membrane of meninges, are the second most common tumors of the central nervous system with an incidence of $20 \%$ of all intracranial tumors and an overall incidence of 2.3/100,000 (1). Meningiomas may be found as intracranial or spinal tumors, but $90 \%$ of meningiomas develop in the brain. The majority of these tumors are benign, and rarely present with histological or clinical features that suggest aggressive potential (2). Both benign and malignant meningiomas are treated with surgical excision, with the goal of gross total resection. Subtotal resection is typically followed by radiation, either fractionated radiotherapy or single-fraction radio-surgery, to extend the progression-free survival of the patient. Fractionated radiotherapy can be applied after recurrence or as the primary treatment when the diagnosis is initially made. Single-fraction radiosurgery could also be the primary treatment for duralbased tumors without rapid growth. Atypical and anaplastic tumors are usually radiated post-operatively, irrespective of the extent of the excision, because of a higher incidence of recurrence $(2,3)$.

Although treated with combined therapy, malignant meningiomas often recur after initial resection and subsequent radiation (4). Moreover, although ionizing radiation causes cell death, it has also the potential to increase cell proliferation and promote long-term resistance of cancer cells to different cytotoxic effects. Sublethal doses cause DNA damage and the production of reactive oxygen species, which in turn, trigger the activation of wild-type p53, ataxia telangiectasia mutated and other regulatory proteins including growth factor receptors like the ERBB family of receptors. 
Subsequently, several signaling pathways, such as MEK1/2/ ERK1/2 and PI3K/AKT, enhance their activity and regulate cell survival, growth and other properties of tumor cells (5). In addition, radiation has been shown to promote the invasiveness of different types of tumor cells through the upregulation of secreted proteases, such as matrix metalloproteinases and plasminogen activators (6-9). Some studies also highlight the potential of radiated cells to induce a more prominent angiogenic effect and implicate the same proteolytic enzymes in this procedure $(6,7)$.

Invasion and angiogenesis are both essential mechanisms that promote and sustain tumor growth and metastasis. Proteases are molecules that have been highly implicated in these tumor biological activities, initially because of their ability to break down components of the extracellular matrix, allowing tumor and endothelial cells to spread. Urokinase plasminogen activator (uPA) and its high affinity receptor (UPAR) are key molecules in tumor invasion and progression. uPA catalyzes the conversion of plasminogen to plasmin, which in turn, exerts strong proteolytic effects including the activation of metalloproteinases and growth factors $(10,11)$. The uPA-uPAR system has been implicated in several tumor processes (e.g., adhesion, migration, proliferation and angiogenesis) through interactions with molecules on the cell surface (e.g., integrins and vitronectin) $(12,13)$, and through activation of intracellular signaling pathways $(14,15)$. Recent studies have shown that radiation increases uPA activity as well as the invasive and angiogenic properties of neuroblastoma cells (6).

Previous studies have demonstrated that meningiomas express high levels of uPA (16). We have shown previously that the downregulation of UPA and UPAR inhibits invasion, angiogenesis and tumor growth in vivo in a variety of tumor types including meningiomas (17-19). Here, we studied the effect of radiation on the malignant meningioma cell line, IOMM-Lee. Our results indicate that radiation increases uPA levels and activity through EGFR, ERK1/2 and p38 signaling molecules and, to a lesser extent, through $\mathrm{pI} 3 \mathrm{~K} /$ AKT. Radiation treatment enhanced invasion, migration and angiogenesis, whereas treatment with siRNA against UPA and uPAR decreased the radiation-induced aggressive properties of IOMM-Lee cells and inhibited tumor growth in vivo.

\section{Materials and methods}

Cell culture, transfection conditions and reagents. The established malignant meningioma cell line IOMM-Lee was kindly provided by Dr Ian E. McCutcheon (University of Texas M.D. Anderson Cancer Center, Houston, TX). Cells were grown in DMEM supplemented with $10 \%$ fetal bovine serum, $100 \mu \mathrm{g} / \mathrm{ml}$ streptomycin and $100 \mathrm{U} / \mathrm{ml}$ penicillin, and maintained in a humidified atmosphere containing $5 \% \mathrm{CO}_{2}$ at $37^{\circ} \mathrm{C}$. The primary antibodies used for this study were antiUPAR (R\&D Systems, Minneapolis, MN), anti-JNK, antiphospho-JNK, anti-p38, anti-phospho-38, anti-ERK, antiphospho-ERK, anti-EGFR (Santa Cruz Biotechnology, Santa Cruz, CA), anti-phospho-EGFR, anti-AKT, anti-phosphoAKT and anti-GAPDH (Cell Signaling, Boston, MA), and HRP-conjugated secondary antibodies (Biomeda, Foster
City, CA). Specific inhibitors of EGFR (AG1478), MEK1/2 (U0126), p38 (SB203580), c-Jun NH2-terminal kinase (JNK) (SP600125), phosphatidylinositol 3-kinase (PI3K) (LY294002), and AKT (AKT inhibitor IV) (Calbiochem, San Diego, CA) were diluted and used as per the manufacturer's instructions.

Construction of siRNA-expressing plasmids. Plasmids were constructed as described previously (17). Briefly, a pcDNA-3 plasmid with a human cytomegalovirus (CMV) promoter was used to construct the siRNA-expressing vectors. The uPA sequence 21 bases long with a 9 base loop region from +346 to +367 (5'-agctTGAGAGCCCTGCTGGCGCGCC $\underline{\text { atatataat }}$ GGCGCGCCAGCAGGGCTCTCA-3'), and with HindIII restriction sites incorporated at the ends, was ligated into the HindIII restriction site of a pcDNA-3 vector plasmid (puPA). Similarly, for the bicistronic construct, the uPAR sequence (5'-gatcCTACAGCAGTGGAGAGCGATT atatataatAATC GCTCTCCACTGCTGTAG-3') with BamHI restriction sites incorporated at the ends was ligated into BamHI restriction site of the puPA vector (pu2). A pcDNA3-scrambled vector with an imperfect sequence, which does not form a perfect hairpin structure, was used to develop the scrambled vector for use as a control (pSV).

Radiation. The RS 2000 Biological Irradiator (Rad Source Technologies, Inc., Boca Raton, FL) X-ray unit, operated at $150 \mathrm{kV} / 50 \mathrm{~mA}$, was used for radiation treatments. Cells were transfected with mock, pSV, puPA or pu2 plasmids using Lipofectamine reagent (Invitrogen, Carlsbad, CA) as per manufacturer's instructions. A single dose of radiation (5 or $10 \mathrm{~Gy}$ ) was given to transfected or control IOMM-Lee cells (in 6-well plates) and tumor spheroids (in 96-well plates).

MTT proliferation assay. Cells were grown in 6-well plates and transfected with pSV, puPA, pu2 or mock (PBS control). Twelve hours after transfection, cells were serum-starved for $6 \mathrm{~h}$, radiated with $10 \mathrm{~Gy}$. Six hours later, cells were trypsinized, counted and seeded at $2 \times 10^{4}$ cells per well in 96well plates (8-wells per treatment group). After the indicated hours of incubation in conditioned medium, $20 \mu 1$ of MTT reagent were added to the cells, followed by another $4 \mathrm{~h}$ of incubation at $37^{\circ} \mathrm{C}$. Acid-isopropanol $(0.04 \mathrm{M} \mathrm{HCl} /$ isopropanol) was added to all wells and mixed vigorously so that the formazan crystals dissolved effectively. Absorbance was measured on microtiter plate reader (Bio-Rad, Model 680 , Hercules, CA) with a test wavelength of $550 \mathrm{~nm}$ and a reference wavelength of $655 \mathrm{~nm}$.

Reverse transcription-PCR analysis. RT-PCR was performed as described previously (19). Cellular RNA was isolated using the Qiagen RNeasy kit, $1 \mu \mathrm{g}$ of RNA was DNasetreated (10 units $/ \mu \mathrm{g}$ of RNA for $1 \mathrm{~h}$ ) and used as a template for the reverse transcription reaction. For first strand cDNA synthesis, $2 \mu \mathrm{g}$ of RNA was used. The following primers were used: uPA sense, 5'-TGCGTCCTGGTCGTGAGCGA-3'; and uPA antisense, 5'-CTACAGCGCTGACACGCTTG-3'; GAPDH sense, 5'-CGGAGTCAACGGATTTGGTCGTAT-3'; and GAPDH antisense, 5'-AGCCTTCTCCATGGTGGTG AAGAC-3'. The PCR conditions were as follows: $95^{\circ} \mathrm{C}$ for 
5 min, followed by 35 cycles of $95^{\circ} \mathrm{C}$ for $1 \mathrm{~min}, 55^{\circ} \mathrm{C}$ for $1 \mathrm{~min}$ and $72^{\circ} \mathrm{C}$ for $1 \mathrm{~min}$. The final extension was at $72^{\circ} \mathrm{C}$ for $5 \mathrm{~min}$. PCR products were resolved on a $1 \%$ agarose gel, visualized, and photographed under UV light.

Fibrinogen zymography. IOMM-Lee cells were transfected with mock, pSV, puPA or pu2 and radiated with 10 Gy as described above. Thirty minutes before radiation, cells were treated with specific inhibitors as per the manufacturer's instructions. Conditioned media were collected at the indicated time points, and zymography was performed to determine uPA activity. Equal amounts of protein samples $(0.5 \mu \mathrm{g})$ were resolved on $10 \%$ SDS-polyacrylamide gel containing plasminogen and fibrinogen, and stained as described previously (17).

Western blot analysis. IOMM-Lee cells were transfected with mock, pSV, puPA or pu2 and radiated with $10 \mathrm{~Gy}$ as described above. Cells were collected and lysed in RIPA buffer [50 mmol/ml Tris-HCl (pH 8.0), $150 \mathrm{mmol} / \mathrm{ml}$ $\mathrm{NaCl}, 1 \%$ IGPAL, $0.5 \%$ sodium deoxycholate, $0.1 \% \mathrm{SDS}$ ] containing $1 \mathrm{mM}$ sodium orthovanadate, $0.5 \mathrm{mM}$ PMSF, $10 \mu \mathrm{g} / \mathrm{ml}$ aprotinin, $10 \mu \mathrm{g} / \mathrm{ml}$ leupeptin and resolved on SDS-PAGE. After overnight transfer onto a PVDF membrane, the blot was blocked with $6 \%$ non-fat dry milk in PBS and $0.1 \%$ Tween-20. The blot was incubated with primary antibody, followed by HRP-conjugated anti-mouse or anti-rabbit secondary antibody. Signals were detected using the ECL system (Amersham Bioscience). Comparable loading of proteins on the gel was verified by re-probing the blots with an antibody specific for the housekeeping gene product, GAPDH.

Spheroid migration. Multicellular IOMM-Lee spheroids were cultured in 96-well ultra low attachment plates. Briefly, $5 \times 10^{4}$ cells per well were seeded and cultured on a shaker at $100 \mathrm{rpm}$ for $48 \mathrm{~h}$ at $37^{\circ} \mathrm{C}$. After single spheroids formed, cells were transfected with mock, pSV, puPA or pu2. Twelve hours after transfection, the spheroids were radiated with $10 \mathrm{~Gy}$, transferred to 8-well chamber slides, and allowed to grow for another $24 \mathrm{~h}$. The results were observed under a light microscope and the migration distance was measured using Image Pro Discovery software.

Matrigel invasion assay. IOMM-Lee cells were transfected with mock, pSV, puPA or pu2 and radiated as described above. After $6 \mathrm{~h}$, cells were trypsinized and $2.5 \times 10^{5}$ cells were placed into matrigel-coated transwell inserts with a $8-\mu \mathrm{m}$ pore size. Cells were allowed to migrate through the matrigel for $24 \mathrm{~h}$. Then, cells in the upper chamber were removed with a cotton swab. The cells that adhered on the outer surface of the transwell insert and had invaded through the matrigel were fixed, stained with Hema-3 (Fisher Scientific), and counted under a light microscope as described previously (17).

In vitro angiogenesis assay. Tumor conditioned mediuminduced microtubule network formation was determined as described previously (17). Meningioma cells were transfected and radiated as described above. Conditioned medium was collected and added to human microvascular endothelial cells (HMEC-1), derived from dermis and provided by Dr Francisco J. Candal (Centers for Disease Control and Prevention, Atlanta, GA, USA), that were seeded the previous day in 96-well plates coated with matrigel. HMEC-1 were incubated overnight and the formation of the microtubule networks was examined using a phase-contrast microscope equipped with a CC camera and evaluated by Discovery Image Pro software. All experiments were repeated at least three times. Results are presented as the means \pm SE of eight fields.

Animal studies. IOMM-Lee cells $\left(5 \times 10^{6}\right)$ were injected subcutaneously into the flank position of 6-8-week old female nude mice. After two weeks, when the tumors reached 4-5 mm in diameter, animals were separated into 4 treatment groups of 10 animals each. Animals were treated on alternate days with intratumoral injections of $\mathrm{pSV}$, puPA or pu2 for a total of four doses $(60 \mu \mathrm{g} / \mathrm{dose})$. Control animals were injected with PBS only (Mock). Between the first and the second injections, and the second and the third injections, 5 animals from each group were radiated with a dose of $5 \mathrm{~Gy}$, each time. During radiation treatment, the entire mouse body except the tumor area was covered with lead sheets so only the tumor would be exposed to the radiation. Subcutaneous tumor growth was measured every 3 days with vernier calipers. Tumor volume was calculated using the formula: $\pi / 6 \mathrm{X}\left(\mathrm{R}_{\max } \times \mathrm{R}_{\min }{ }^{2}\right)$, where $\mathrm{R}=$ tumor diameter. Regression in subcutaneous tumor growth was followed for up to 3 weeks. Mice were euthanized when the tumor diameter in control mice measured between 11 and $12 \mathrm{~mm}$. Subcutaneous tumors were removed and fixed in buffered formaldehyde. Paraffinembedded subcutaneous tumor sections were stained with $\mathrm{H} \& \mathrm{E}$ using standard histological techniques.

For immunohistochemical analyses for uPA and uPAR or vessel staining, the sections were deparaffinized in xylene and rehydrated through graded ethanol. Antigen retrieval was carried out by treating sections with $0.1 \%$ Triton $\mathrm{X}-100$ and $0.1 \%$ sodium citrate buffer $(\mathrm{pH} 6.0)$ for $20 \mathrm{~min}$. Following quenching of endogenous peroxidase activity and blocking of non-specific binding, sections were incubated overnight at $4^{\circ} \mathrm{C}$ with an anti-uPA, anti-uPAR or CD31 antibody (endothelial surface marker) (1:100 dilution). Next, sections were treated with HRP-conjugated secondary antibody (1:200 dilution) for $30 \mathrm{~min}$ at RT. Immunolocalization was accomplished by exposing sections to $0.05 \%$ 3,3'-diaminobenzidine tetrahydrochloride (DAB) as the chromogen. Non-specific IgG (Mouse IgG) was used in the place of primary antibody as a negative control. The slides were counterstained with Mayer's hematoxylin and mounted. All microscopy studies were performed using a microscope attached to a CC camera and set to auto.

Statistical analysis. All data are presented as means \pm standard errors (SE) of at least three independent experiments, each performed at least in triplicate. One-way analysis of variance (ANOVA) combined with the Tukey post-hoc test of means were used for multiple comparisons in cell culture experiments. Statistical differences are presented at probability levels of $\mathrm{p}<0.05, \mathrm{p}<0.01$ and $\mathrm{p}<0.001$. 
A
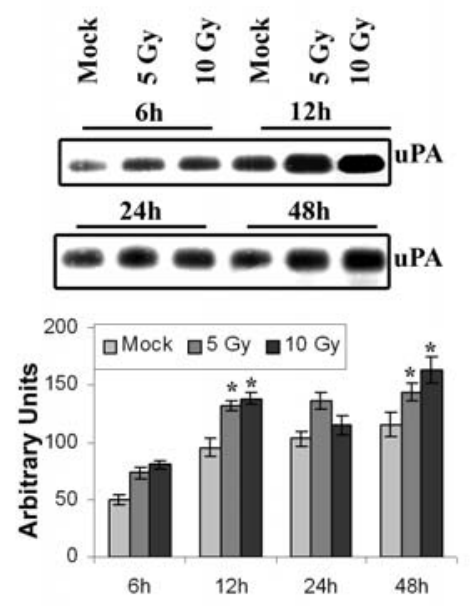

B
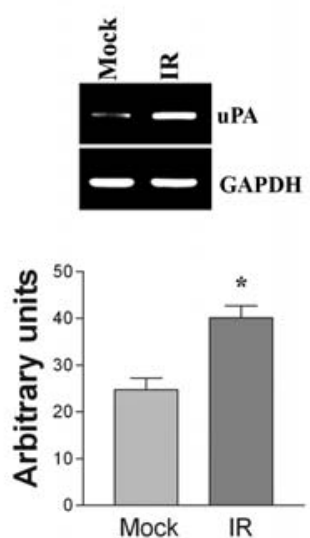
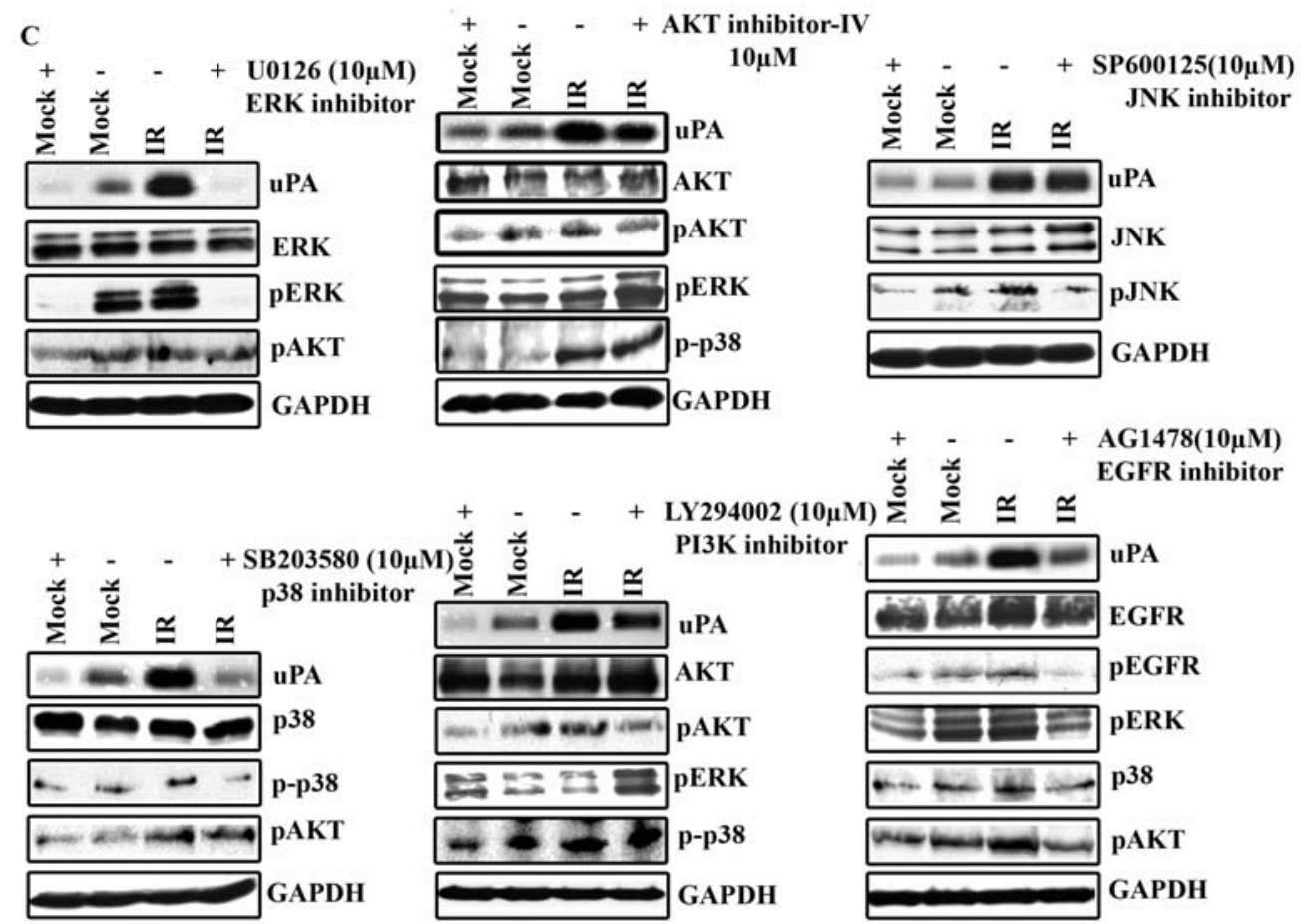

Figure 1. Radiation increases the expression uPA and other signaling pathway molecules in IOMM-Lee cells. (A) Fibrinogen zymography using the conditioned medium from IOMM-Lee cell cultures radiated with 5 or $10 \mathrm{~Gy}$ and controls for the indicated time-points. Equal amounts of total protein $(0.5 \mu \mathrm{g})$ were resolved for each sample. Mean densitometric values \pm SEM were calculated and plotted as a histogram. *Statistically different compared to respective control and radiated groups $(\mathrm{p}<0.05)$. (B) RT-PCR analysis for uPA expression. Total RNA was extracted from IOMM-Lee cells incubated in serum-free medium for $6 \mathrm{~h}$ after cells were radiated (with $10 \mathrm{~Gy}$ ) or not irradiated (mock). GAPDH was used as a loading control. Mean densitometric values \pm SEM were calculated and plotted as a histogram. "Statistically different compared to control and radiated groups (p<0.05). (C) EGFR, through ERK1/2 and p38 and to a lesser extent, $\mathrm{pI} 3 \mathrm{~K} / \mathrm{AKT}$, mediates radiation-induced uPA in IOMM-Lee cells. Fibrinogen zymography of conditioned medium, and Western blot analysis of cell lysates from IOMM-Lee cells. Cells were treated with specific inhibitors of JNK (SP600125) (15 $\mu$ M), ERK1/2 (U0126) (10 $\mu$ M), p38 (SB203580) $(10 \mu \mathrm{M})$, AKT (AKT inhibitor IV) $(10 \mu \mathrm{M})$, pI3K (LY294002) $(20 \mu \mathrm{M})$ and EGFR (AG1478) (10 $\mu \mathrm{M})$ for 30 min before they were radiated with $10 \mathrm{~Gy}$ and subsequently incubated for $6 \mathrm{~h}$ in serum-free medium. Conditioned medium was collected and $0.5 \mu \mathrm{g}$ of total protein was used for fibrinogen zymography to detect uPA activity. Equal amounts of total proteins from cell extracts were resolved by SDS-PAGE and probed with antibodies against phosphorylated JNK, ERK1/2, p38, AKT, EGFR and total JNK1/2, ERK1/2, p38, AKT and EGFR. GAPDH was used as a loading control. Experiments were repeated at least three times.

\section{Results}

Radiation increases activity and quantity of $u P A$. Radiation is shown to enhance invasiveness of several types of tumor cells via stimulating the expression of proteolytic molecules, including uPA $(7-9,20)$. To determine whether radiation increased uPA expression in IOMM-Lee cells, we studied the dose- and time-dependency of uPA expression and activity in the initial experiments. Irradiated IOMM-Lee cells that received different doses of $\mathrm{x}$-rays were incubated for different time-points. Conditioned medium was analyzed for uPA activity using fibrinogen zymography. As shown in Fig. 1A, uPA activity increased with dose (5 vs. 10 Gy of radiation) and with time (6 vs. $12 \mathrm{~h}$ ); uPA decreased thereafter. Densitometric analysis of the fibrin zymogram demonstrated that after $6 \mathrm{~h}$, uPA activity was significantly increased 
A

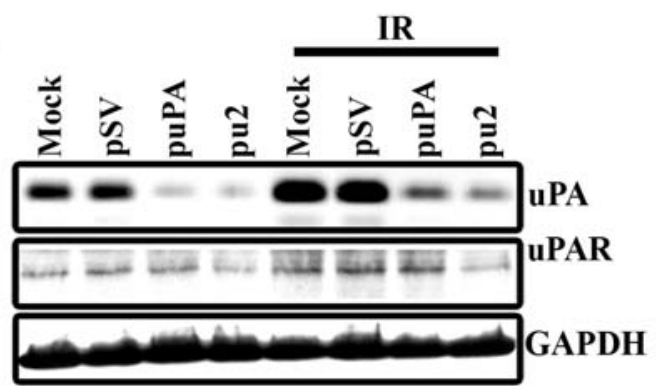

B

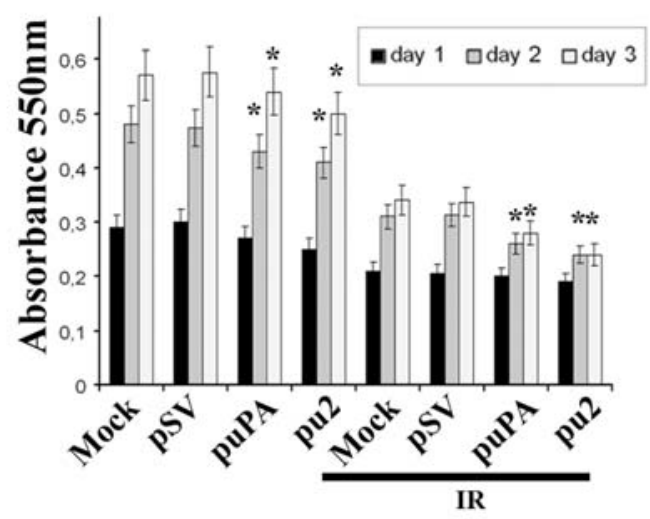

Figure 2. puPA and pu2 siRNA inhibit radiation-induced uPA and UPAR. (A) Cells were transfected with mock, pSV, puPA or pu2 and $18 \mathrm{~h}$ later, cells were radiated with $10 \mathrm{~Gy}$ and incubated in serum-free medium for $6 \mathrm{~h}$. Conditioned medium $(0.5 \mu \mathrm{g})$ was used for fibrinogen zymography and equal amounts of total protein from cell extracts were resolved on SDS-PAGE and probed with anti-uPAR primary antibody. GAPDH was used as a loading control. (B) MTT proliferation assay. Cells were transfected with the plasmids and irradiated as described above. Six hours later, cells were trypsinized and $2 \times 10^{4}$ cells were seeded on 96-well plates (eight wells per treatment). Cell proliferation was measured at 24, 48 and $72 \mathrm{~h}$ as described in Materials and methods. The bars represent the means $\pm \mathrm{SE}$ of three different experiments. *Statistically different compared to control and puPA or pu2 treated groups or IR+control and IR + puPA or IR + pu2 groups $(\mathrm{p}<0.05)$.

as compared to the control (mock). After $12 \mathrm{~h}$, uPA activity was further increased and uPA activity reached saturation point at $24 \mathrm{~h}$. Based on these results, we chose a dose of $10 \mathrm{~Gy}$ and a 6-h time-point for the remaining experiments. To quantify mRNA transcripts levels, we performed RTPCR analysis, which revealed that radiation induced uPA expression at the transcriptional level (Fig. 1B). The quantification of mRNA levels using densitometric analysis showed a $65 \%$ increase of uPA levels in irradiated IOMM-Lee cells (Fig. 1B).

Radiation-induced UPA secretion via ERK1/2 and p38 pathways. uPA expression is mediated through growth factor receptors like EGFR, and signaling pathways like ERK1/2, p38 MAP kinase and pI3K-AKT (21-24). To determine which signaling pathways were affected by radiation-induced uPA expression, we performed Western blot analysis using cell lysates from irradiated IOMM-Lee cells. As shown in Fig. 1C, radiation at 10 Gy increased phosphorylation of ERK1/2, p38 and JNK significantly, but not phosphorylation of AKT. At a concentration of $10 \mu \mathrm{M}$, the specific inhibitor of ERK1/2, U0126, inhibited both basal and radiation-induced
uPA secretion. A similar effect was observed with a p38 inhibitor, SB203580. The PI3K inhibitor (LY294002) and AKT inhibitor (AKT inhibitor IV) only partially reduced radiation-induced uPA induction. In contrast, SP600125, a JNK inhibitor, did not have any effect on uPA expression levels (Fig. 1C). Inhibition of the PI3K/AKT signaling pathway increased the phosphorylation status of ERK $1 / 2$, which is consistent with previous studies (25). However, uPA expression was not significantly inhibited after pI3K/AKT blockade (Fig. 1C). Taken together, these results indicate that radiation-induced uPA expression is mediated by the ERK1/2 and p38 signaling pathways, and partially through the PI3K/AKT signaling pathway.

Radiation induces $u P A$ expression via epidermal growth factor receptors (EGFR). Because meningioma tumor cells express distinct levels of EGFRs $(26,27)$, we next examined whether EGFR is implicated in radiation-induced uPA secretion in IOMM-Lee cells. Indeed, inhibition of EGFR phosphorylation by a specific inhibitor, AG1478, decreased both basal and radiation-induced uPA levels in a dosedependent manner (data not shown for the dose-dependent effect), and also reduced phosphorylation of downstream molecules ERK1/2, p38 and AKT (Fig. 1C). Collectively, the results indicate that radiation-induced uPA expression is mediated via the EGFR/MEK/ERK and EGFR/MEK/p38 pathways in the IOMM-Lee meningioma cell line.

puPA and pu2 downregulate radiation-induced $u P A$ activity and $U P A R$ levels. To determine the effect of the downregulation of uPA and UPAR levels in irradiated cells using siRNA constructs for uPA (puPA) and for both uPA and UPAR (pu2), we transfected IOMM-Lee cells with mock (PBS), pSV, puPA or pu2 for $48 \mathrm{~h}$ and then cells were radiated with $10 \mathrm{~Gy}$. Six hours later, conditioned medium was collected and fibrin zymography performed to assess uPA activity and Western blotting performed from cell lysates for uPAR levels. As shown in Fig. 2A, we observed significant inhibition of UPA activity in both irradiated and non-irradiated samples, transfected with either puPA or pu2. In addition, cell lysates of pu2-transfected IOMM-Lee cells showed significant reduction of uPAR expression levels (Fig. 2A) in both irradiated and non-irradiated conditions.

puPA and pu2 decrease cell proliferation. To determine the effect of radiation-induced uPA on cell proliferation of puPA- or pu2-treated IOMM-Lee cells, we performed the MTT proliferation assay. puPA- and pu2-treated cells showed significant reductions in cell proliferation after radiation as compared to the controls and non-radiated cells (Fig. 2B). The reduction in cell proliferation in irradiated cells vs. non-irradiated cells may be due to DNA damage caused by $\mathrm{x}$-ray radiation, which might enhance delay in progression of the cell cycle.

puPA and pu2 decrease radiation-induced migration and invasion. Recent studies have highlighted the ability of radiation to enhance tumor cell migration and invasion in cells that received a sublethal dose through the upregulation of proteolytic molecules (28). With the significant increase 
A Mock

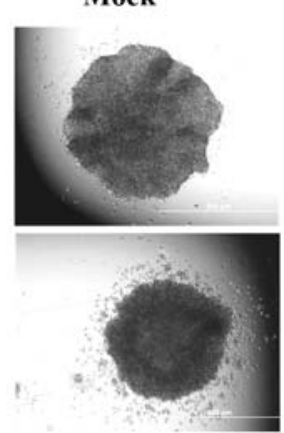

pSV
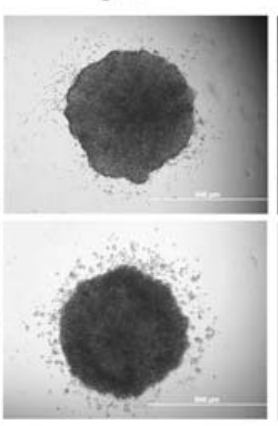

pSV

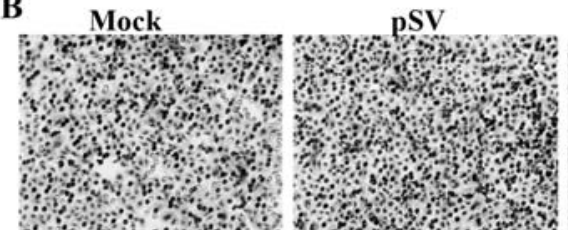

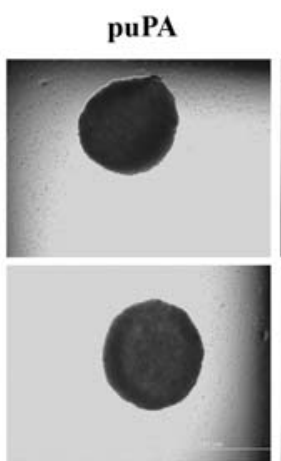

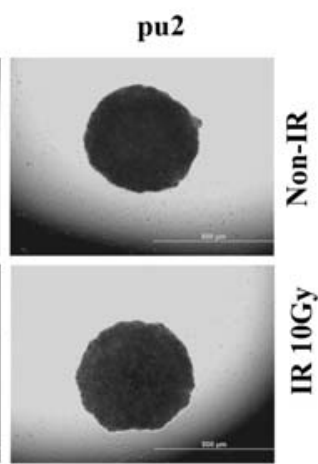

\section{$\stackrel{1}{1}$}

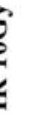

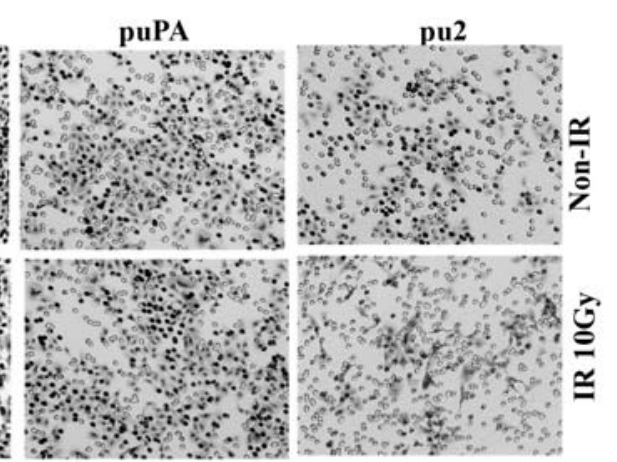

C

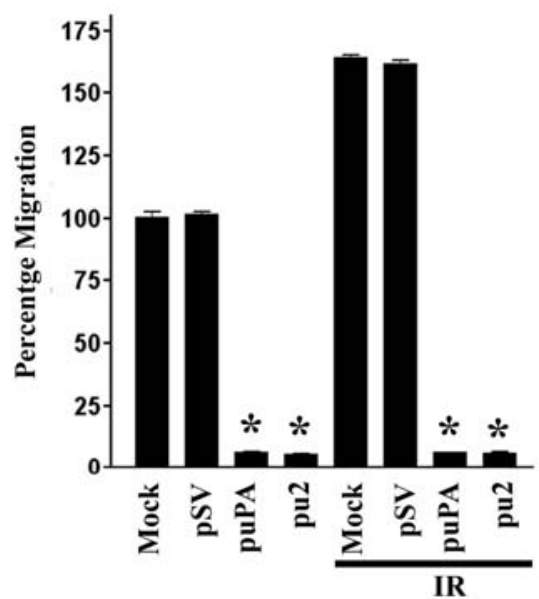

D

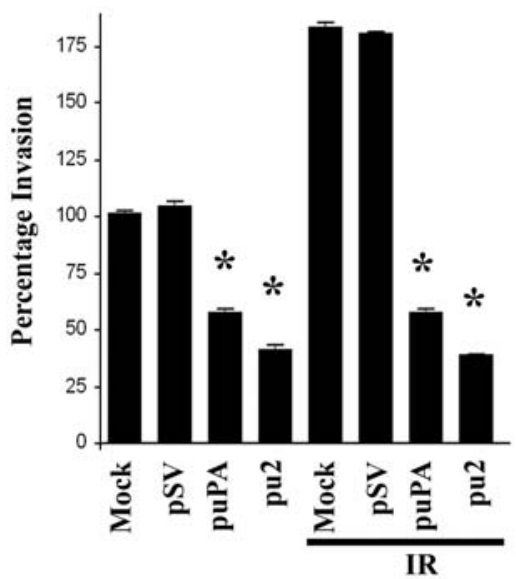

Figure 3. Inhibition of radiation-induced migration from tumor spheroids and invasion through matrigel. (A) After IOMM-Lee cells formed single spheroids, they were transfected with mock, pSV, puPA or pu2 and transferred to 8-well chamber slides and irradiated with 10 Gy. Spheroids were allowed to grow in serum-free medium for $24 \mathrm{~h}$ and were photographed under a light microscope. (B) Matrigel invasion assay of IOMM-Lee cells transfected for $24 \mathrm{~h}$ and then radiated with $10 \mathrm{~Gy}$. After $6 \mathrm{~h}, 2.5 \times 10^{5}$ cells were plated on matrigel-coated transwell inserts. Invasion was allowed for $24 \mathrm{~h}$, and then inserts were stained with HEMA stain. Cells that had migrated through the matrigel were counted and photographed under a light microscope. (C) Migration from the spheroids was measured using Image-Pro Discovery software. Average values of three separate experiments are shown and bars represent the means \pm SE. * Statistically different compared to control and puPA or pu2 treated groups or IR+control and IR + puPA or IR + pu2 groups (p<0.01). (D) Quantification of invading cells. The bars represent the means \pm SE of three different experiments. ${ }^{*}$ Statistically different compared to control and puPA or pu2 treated groups or IR+control and IR + puPA or IR + pu2 groups $(\mathrm{p}<0.01)$.

of uPA levels in the conditioned medium of irradiated IOMMLee cells, we examined the effect of radiation on cell migration and invasion. We noticed that radiation at a dose of 10 Gy significantly enhanced spheroid migration by $\sim 65 \%$ compared to the non-irradiated controls (Fig. 3A and C). However, transfection with puPA and pu2 significantly inhibited migration by 79.66 and $80.47 \%$, respectively as compared with the mock and pSV controls (Fig. 3C), but in irradiated cells the migration was inhibited with puPA and pu2 $\sim 94$ and $\sim 95 \%$ respectively compared with mock and pSV controls.

We also observed a significant inhibition of the number of cells that invaded through transwell inserts coated with matrigel in both puPA- and pu2-treated IOMM-Lee cells (Fig. 3B). To facilitate the comparison of the relative invasiveness between irradiated and non-irradiated cells, all values were normalized to the percentage invasion of mock control cells, which was taken as $100 \%$ (Fig. 3D). 

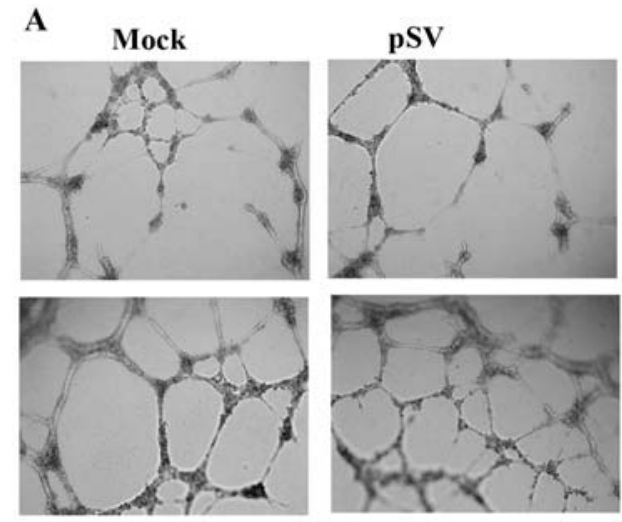

B

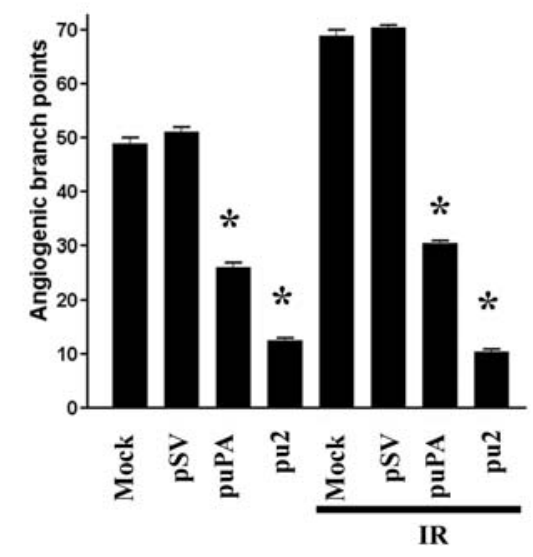

Quantification of the cells that invaded through the transwell inserts showed that irradiated IOMM-Lee cells invaded $\sim 75 \%$ more than non-irradiated cells (Fig. 3D). Treatments with puPA and pu2 significantly decreased invasion as compared with the irradiated mock and pSV controls (Fig. 3D). Notably, irrespective of radiation, treatments with puPA and pu2 decreased invasion of cells by $\sim 50$ and $~ 35 \%$, respectively.

puPA and pu2 inhibit radiation-induced capillary network formation in $H M E C$-1. Proteolytic molecules that are induced by radiation, such as UPA, have been implicated in tumor angiogenesis (29). Because UPA and UPAR have been reported to regulate angiogenesis, we next assessed the effect of pu2 on radiation-induced angiogenesis. To evaluate tumorinduced vessel formation in an in vitro co-culture system, conditioned medium from irradiated IOMM-Lee cells was added to HMEC-1 monolayers. Radiation treatment enhanced capillary-like network formation by $30 \%$, whereas pre-treatment of tumor cells with puPA and pu 2 significantly blocked the angiogenic process as compared to the tumor conditioned medium from irradiated and non-irradiated mock and pSVtransfected IOMM-Lee cells (Fig. 4).

Quantification of the branch points per field was extremely low in pu2-transfected co-cultures when compared with mock and pSV-transfected cells. The effect was $<20 \%$ and $<26 \%$ less in irradiated and non-irradiated pu2-transfected co-cultures, respectively compared to control co-cultures (Fig. 4).

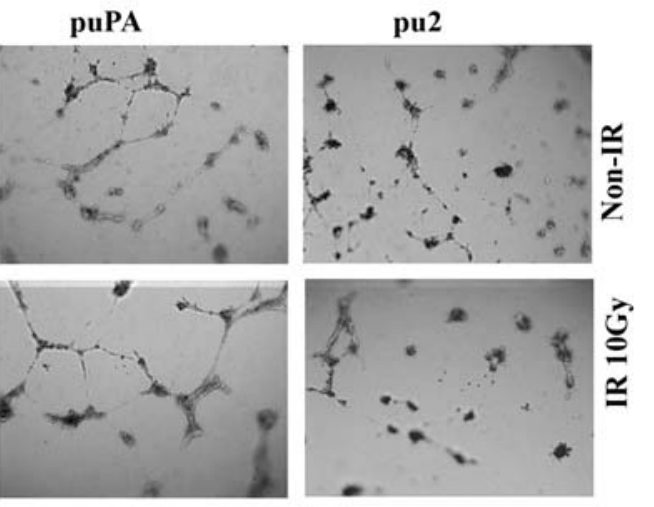

Figure 4. Downregulation of uPA and UPAR decreases radiation-induced tumor angiogenesis. (A) Conditioned medium from IOMM-Lee cells, which were transfected with mock, pSV, puPA or pu2 and subsequently radiated with $10 \mathrm{~Gy}$, was added to human microvascular endothelial cells (HMEC-1) pre-seeded on matrigel-coated 96-well plates. After overnight incubation, HMEC-1 were observed for capillary-like network formation and photographed under a light microscope. (B) Angiogenic effect was measured by counting the relative branch-points. Bars represent the means \pm SE of three different experiments. *Statistically different compared to control and puPA or pu 2 treated groups or IR+control and IR + puPA or IR + pu2 groups $(\mathrm{p}<0.01)$.

puPA and pu2 treatments plus radiation suppress tumor growth in vivo. To study the in vivo effect of RNA interference and radiation simultaneously, we injected the human meningioma cell line IOMM-Lee to generate subcutaneous tumors in athymic nude mice as described in Materials and methods. Fig. 5A shows that drastic reduction in tumor growth of the radiated xenografts in puPA- and pu2-treated animals as compared to mock and pSV-treated controls. pu2 treatment along with radiation had a synergistic effect on preventing tumor progression (Fig. 5A). Further, pu2 treatment alone inhibited tumor growth compared to puPA nonirradiated groups and controls. Tumor volume quantification indicated a significant reduction of 75 and $95 \%$ in tumor size in pu2-treated non-irradiated and irradiated animals $(\mathrm{P}<0.01)$ respectively, as compared with mock and pSV-treated control animals (Fig. 5B).

puPA and pu2 treatment inhibits expression of $u P A, u P A R$, and CD31 in vivo. To correlate the in vitro results with in vivo experiments, we analyzed expression of UPA, uPAR and CD31 in tissue sections of IOMM-Lee meningioma subcutaneous tumors raised in nude mice. Immunohistochemical analysis revealed that both uPA and UPAR were expressed more in the radiated as well as mock and pSVtreated mice xenografts. In contrast, siRNA treatment specifically inhibited the expression of UPA and UPAR in vivo (Fig. 6). Sections from mice treated with mock and pSV exhibited high expression levels of UPA and UPAR and, as expected, radiation treatment increased UPA and UPAR expression further. In contrast, the decreased immunoreactivity of UPA and UPAR in tumors from the puPA- and pu2-treated mice serves as evidence that the downregulation of uPA and its receptor reduce tumor growth (Fig. 6, middle panel). To determine whether the inhibition of uPA and uPAR affected angiogenesis, the sections were stained with 


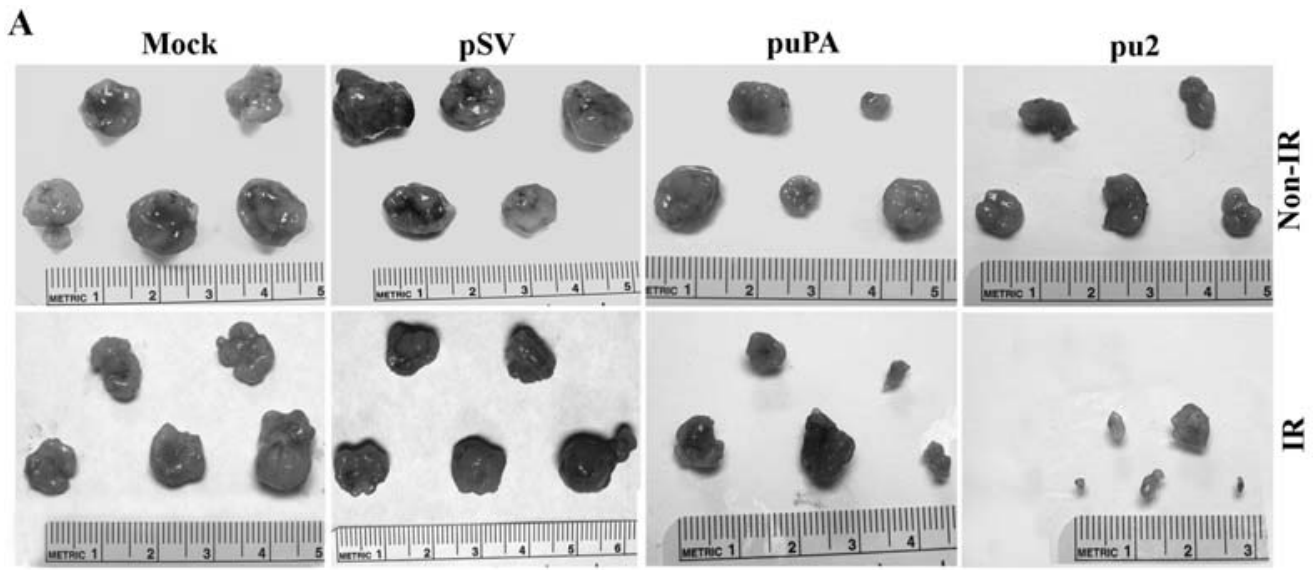

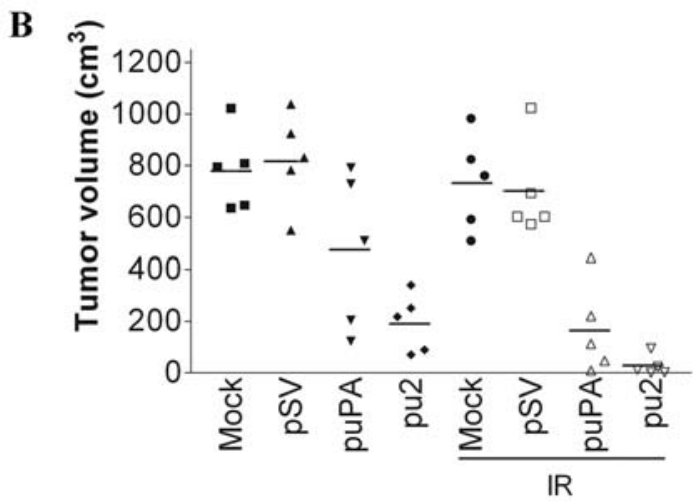

CD31 antibody (Fig. 6, bottom panel), which allows for the visualization of blood vessels. A significant reduction of stained vessels was observed in tumor sections from puPAand pu2-treated animals (both irradiated and non-irradiated groups) when compared with tumor sections from mock and $\mathrm{pSV}$ treated groups. Thus, targeting uPA and uPAR expression along with radiation effectively prevented the growth of meningioma xenograft tumors.
Figure 5. puPA and pu2 along with radiation suppress subcutaneous tumor growth in athymic nude mice. (A) IOMM-Lee cells $\left(5 \times 10^{6}\right)$ were injected subcutaneously into athymic nude mice. After tumors reached 4-5 mm in diameter, mice were treated intratumorally with mock or with 4 doses (60 $\mu \mathrm{g} / \mathrm{dose})$ of $\mathrm{pSV}$, puPA or pu2. Between the injections, animals were irradiated twice with 5 Gy as described in Materials and methods. Three weeks after treatments were completed, animals were sacrificed and tumors removed, measured and photographed. (B) Tumor volume was measured as described in Materials and methods. Tumor volumes are shown as symbols and the average values of all tumor volume is shown as horizontal solid line ( 5 animals per treatment group) $(\mathrm{p}<0.01)$.

\section{Discussion}

Radiation is widely used for the treatment of meningiomas even after gross total resection and especially after subtotal excision $(2,3)$. Although radiation is an established treatment for several types of tumors, recent studies indicate that radiation may also increase the invasive potential of cells that received sublethal doses (7-9). Here, we studied the effect of radiation in combination with UPA and UPAR downregulation on the malignant meningioma cell line IOMM-Lee. During the preliminary experiments, we noticed that uPA activity

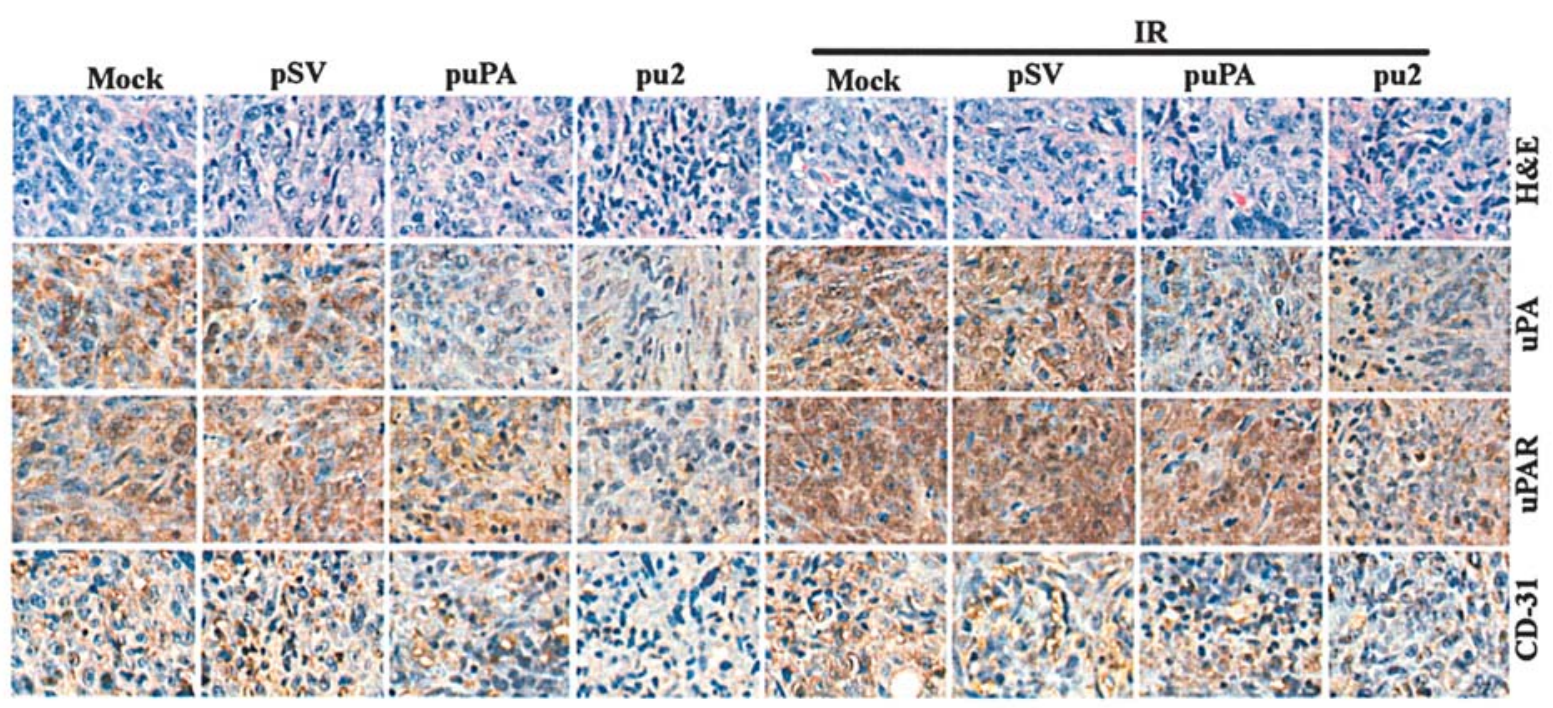

Figure 6. puPA or pu2 downregulate radiation-induced uPA, uPAR and CD31 in tumor xenografts. Hematoxylin and eosin staining of sections from IOMMLee xenograft tumors (top) and immunohistochemical analyses for uPA (second from top), uPAR (second from bottom) and CD31 (bottom) using specific antibodies for UPA, uPAR and CD31. 
increases as a cellular response to sublethal doses of radiation. Next, we elucidated the possible role of signaling pathways, which are commonly activated in irradiated cells, in uPA regulation. Subsequently, we used RNA interference to downregulate UPA alone or in combination with its receptor UPAR, in irradiated and non-irradiated cells. Our results show that siRNA against uPA and uPAR inhibited invasion and tumorinduced angiogenesis of irradiated IOMM-Lee cells, and also suppressed tumor growth in vivo. In essence, radiation sensitization enhanced siRNA against uPA and UPAR in a synergistic manner.

Because sublethal doses of radiation are capable of activating growth factor receptors and pro-survival signaling pathways, they have the potential to augment cell proliferation and resistance $(5,8)$. Radiation activates auto-phosphorylation of EGFR and enhances the activity of protein tyrosine kinase without ligand interaction. In turn, the auto-phosphorylation of EGFR triggers downstream pathways. Radiation causes a rapid reactive oxygen species-dependent activation of ERBB family and other tyrosine kinases, leading to the activation of RAS proteins and multiple protective downstream signaling pathways like AKT, ERK1/2 and JNK, which alter transcription factor function and the apoptotic threshold of cells (5). Further, cell growth regulating pathways, such as RAF-1, MEK1/2-ERK1/2, p38 and pI3K-AKT, are shown to be downstream responses of radiation-induced activation of EGFR family receptors $(8,29)$. There are reports showing that the level of EGFR is associated with degree of radio-resistance $(8,30)$. In the current study, Western blots using phosphospecific antibody confirmed that radiation enhances phosphorylated signals of ERK, JNK, p38, and AKT, as well as auto-phosphorylation of EGFR. This protein phosphorylation was inhibited by pre-treatment with each specific inhibitor in IOMM-Lee cells (Fig. 1C).

UPAR is associated with integrins and is known to mediate cellular motility via extracellular matrix components such as vitronectin (31). In addition to integrin-mediated signaling pathways, uPAR is associated with other signaling pathways in variety of tumor cells (21-24). In this study, radiation augmented phosphorylation of p38, ERK1/2 and JNK MAP kinases. However, both a p38 MAP kinase inhibitor and an ERK1/2 inhibitor, but not a SAPK/JNK inhibitor, inhibited radiation-induced uPA expression. These results confirm that radiation-induced uPA expression is mediated through the EGFR/p38 and EGFR/ERK-dependent MAP kinase pathways.

Cell migration, defined as locomotion of a cell over an extracellular matrix (ECM) substratum, involves extension and binding of integrins and other adhesion receptors to their ECM ligands. The interaction of UPA with UPAR at the invading front of the cells initiates cellular events that are mediated by integrins. Binding of UPA to UPAR activates plasmin, which in turn, degrades the ECM and enables cell migration. The uPA-uPAR system is known to interact with several molecules on the cell surface (e.g., integrins, vitronectin, caveolin and $\mathrm{G}$ protein-coupled receptor) and also to be involved in other cell signaling processes $(32,33)$. As a result of this cascade, various growth factors are induced (e.g., bFGF, VEGF, HGF), which in turn, can upregulate uPA and UPAR and act as positive feedback $(34,35)$. Furthermore, the expression of both UPA and UPAR correlates with invasive cancer cell phenotype and poor prognosis (36). Several reports have correlated the invasive and metastatic potential of tumor cells with activity and expression of uPA in different cancer cell lines $(11,36,37)$. In addition, studies have shown that sublethal doses of radiation promote migration and invasiveness in glioma cell line (28). In the present study, as shown by Western blotting and zymography, transient transfection with pu2 decreased endogenous levels of uPAR expression and uPA activity in IOMM-Lee cells, with or without radiation. Moreover, the migration and invasion studies showed slight migration or invasion of cells from spheroids transfected with pu2, with or without radiation.

Angiogenesis, the process by which new branches grow from existing vessels, requires the degradation of the vascular basement membrane and remodeling of the ECM to allow endothelial cells to migrate and invade into the surrounding tissue. As such, the uPA-uPAR system plays a central role in cell migration, adhesion and angiogenesis (27). Because migration and invasion are the initial steps for angiogenesis, we examined whether radiation inducing angiogenesis. In our in vitro experiments on HMEC-1 capillary network formation, the conditioned medium from irradiated cells increased angiogenesis in comparison to non-irradiated cells and angiogenesis was inhibited in tumor conditioned medium either from puPA/pu2 treated alone or in combination with radiation treated IOMM-Lee cells, compared to mock and pSV treated controls.

Our result suggested that the downregulation of both UPA and uPAR is more effective in inhibiting migration, invasion and angiogenesis than the downregulation of either of the molecules alone. These results confirm our previous studies in which uPA and UPAR downregulation inhibited invasion, migration, angiogenesis and survival in different cancer cells (17-19,38-41).

Because UPA and UPAR are frequently overexpressed in many cancers, uPA is believed as an important target molecule for cancer therapy. Furthermore, many studies of clinical tumor samples have correlated tumor progression with high levels of uPA expression and, in some cases, poor patient post-operative survival $(42,43)$. In vivo therapies that target UPA or UPAR in various cancers have been shown to suppress tumor growth $(44,45)$. Resnati et al $(33)$ observed increased metastatic dissemination along with uPAR overexpression in human melanoma xenografts after subcurative radiation treatment. Here, we show that siRNA can effectively suppress UPA-uPAR expression in IOMM-Lee subcutaneous tumors (Fig. 5). Furthermore, the in vivo treatment of pre-established subcutaneous tumors with siRNA demonstrated an almost complete inhibition of tumor growth, whereas only a partial reduction was observed with siRNA against uPA. However, immunohistological analysis for UPA and UPAR in puPA- or pu2-treated xenograft tumor sections of radiated and nonradiated mice showed a relationship with reduction in tumor size. Immunoreactivity of CD31, an endothelial cell marker, was reduced in tumor xenografts treated with either puPA or pu2, indicating a reduction of tumor angiogenesis. Notably, the combination of radiation and siRNA for UPA and UPAR demonstrated the most prominent suppression of angiogenesis, indicating a possible synergistic effect. 
Once again, these results show that the simultaneous downregulation of UPA and UPAR is more effective in inhibiting migration, invasion and angiogenesis than the downregulation of either molecule alone. The present study goes further and shows that the simultaneous downregulation of UPA and UPAR decreases even radiation-induced migration, invasion and angiogenesis in IOMM-Lee cells in vitro and suppresses tumor growth in subcutaneous xenograft tumors in vivo. In conclusion, the combined use of RNAi-based gene therapy and radiation has great potential for the treatment of meningiomas and possibly other brain tumors.

\section{Acknowledgments}

We thank Noorjehan Ali for technical assistance, Shellee Abraham for manuscript preparation, and Diana Meister and Sushma Jasti for manuscript review. This research was supported by National Cancer Institute Grant CA 75557, CA 92393, CA 95058, CA 116708, N.I.N.D.S. NS47699, NS57529 and NS61835, and Caterpillar, Inc., OSF Saint Francis, Inc., Peoria, IL (to J.S.R.).

\section{References}

1. Bondy M and Ligon BL: Epidemiology and etiology of intracranial meningiomas: a review. J Neurooncol 29: 197-205, 1996.

2. Modha A and Gutin PH: Diagnosis and treatment of atypical and anaplastic meningiomas: a review. Neurosurgery 57: 538-550, 2005.

3. Goldsmith B and McDermott MW: Meningioma. Neurosurg Clin N Am 17: 111-120, 2006.

4. Ko KW, Nam DH, Kong DS, Lee JI, Park K and Kim JH: Relationship between malignant subtypes of meningioma and clinical outcome. J Clin Neurosci 14: 747-753, 2007.

5. Valerie K, Yacoub A, Hagan MP, Curiel DT, Fisher PB, Grant S and Dent P: Radiation-induced cell signaling: inside-out and outside-in. Mol Cancer Ther 6: 789-801, 2007.

6. Jadhav U and Mohanam S: Response of neuroblastoma cells to ionizing radiation: modulation of in vitro invasiveness and angiogenesis of human microvascular endothelial cells. Int $\mathbf{J}$ Oncol 29: 1525-1531, 2006.

7. Kaliski A, Maggiorella L, Cengel KA, Mathe D, Rouffiac V, Opolon P, Lassau N, Bourhis J and Deutsch E: Angiogenesis and tumor growth inhibition by a matrix metalloproteinase inhibitor targeting radiation-induced invasion. Mol Cancer Ther 4: 1717-1728, 2005.

8. Park CM, Park MJ, Kwak HJ, Lee HC, Kim MS, Lee SH, Park IC, Rhee $\mathrm{CH}$ and Hong SI: Ionizing radiation enhances matrix metalloproteinase-2 secretion and invasion of glioma cells through Src/epidermal growth factor receptor-mediated p38/ Akt and phosphatidylinositol 3-kinase/Akt signaling pathways. Cancer Res 66: 8511-8519, 2006.

9. Zhai GG, Malhotra R, Delaney M, Latham D, Nestler U, Zhang M, Mukherjee N, Song Q, Robe P and Chakravarti A: Radiation enhances the invasive potential of primary glioblastoma cells via activation of the Rho signaling pathway. J Neurooncol 76: 227-237, 2006.

10. Murphy G, Atkinson S, Ward R, Gavrilovic J and Reynolds JJ: The role of plasminogen activators in the regulation of connective tissue metalloproteinases. Ann NY Acad Sci 667: 1-12, 1992.

11. Rao JS: Molecular mechanisms of glioma invasiveness: the role of proteases. Nat Rev Cancer 3: 489-501, 2003.

12. Wei Y, Waltz DA, Rao N, Drummond RJ, Rosenberg S and Chapman HA: Identification of the urokinase receptor as an adhesion receptor for vitronectin. J Biol Chem 269: 32380-32388, 1994.

13. Wei Y, Lukashev M, Simon DI, Bodary SC, Rosenberg S, Doyle MV and Chapman HA: Regulation of integrin function by the urokinase receptor. Science 273: 1551-1555, 1996.

14. Chandrasekar N, Mohanam S, Gujrati M, Olivero WC, Dinh DH and Rao JS: Downregulation of uPA inhibits migration and PI3k/ Akt signaling in glioblastoma cells. Oncogene 22: 392-400, 2003.
15. Jo M, Thomas KS, Somlyo AV, Somlyo AP and Gonias SL: Cooperativity between the Ras-ERK and Rho-Rho kinase pathways in urokinase-type plasminogen activator-stimulated cell migration. J Biol Chem 277: 12479-12485, 2002.

16. Siddique K, Yanamandra N, Gujrati M, Dinh D, Rao JS and Olivero W: Expression of matrix metalloproteinases, their inhibitors, and urokinase plasminogen activator in human meningiomas. Int J Oncol 22: 289-294, 2003.

17. Gondi CS, Lakka SS, Yanamandra N, Siddique K, Dinh DH, Olivero WC, Gujrati M and Rao JS: Expression of antisense UPAR and antisense UPA from a bicistronic adenoviral construct inhibits glioma cell invasion, tumor growth and angiogenesis. Oncogene 22: 5967-5975, 2003.

18. Kondraganti S, Gondi CS, McCutcheon I, Dinh DH, Gujrati M, Rao JS and Olivero W: RNAi-mediated downregulation of urokinase plasminogen activator and its receptor in human meningioma cells inhibits tumor invasion and growth. Int $\mathrm{J}$ Oncol 28: 1353-1360, 2006.

19. Pulukuri SM, Gondi CS, Lakka SS, Jutla A, Estes N, Gujrati M and Rao JS: RNA Interference-directed knockdown of urokinase plasminogen activator and urokinase plasminogen activator receptor inhibits prostate cancer cell invasion, survival, and tumorigenicity in vivo. J Biol Chem 280: 36529-36540, 2005.

20. Rofstad EK, Mathiesen B and Galappathi K: Increased metastatic dissemination in human melanoma xenografts after subcurative radiation treatment: radiation-induced increase in fraction of hypoxic cells and hypoxia-induced up-regulation of urokinase-type plasminogen activator receptor. Cancer Res 64: 13-18, 2004.

21. Das R, Mahabeleshwar GH and Kundu GC: Osteopontin induces AP-1-mediated secretion of urokinase-type plasminogen activator through c-Src-dependent epidermal growth factor receptor transactivation in breast cancer cells. J Biol Chem 279: 11051-11064, 2004

22. Dunn SE, Torres JV, Oh JS, Cykert DM and Barrett JC: Upregulation of urokinase-type plasminogen activator by insulinlike growth factor-I depends upon phosphatidylinositol-3 kinase and mitogen-activated protein kinase kinase. Cancer Res 61: 1367-1374, 2001

23. Niiya M, Niiya K, Shibakura M, Asaumi N, Yoshida C, Shinagawa K, Teshima T, Ishimaru F, Ikeda $K$ and Tanimoto $M$ : Involvement of ERK1/2 and p38 MAP kinase in doxorubicininduced uPA expression in human RC-K8 lymphoma and NCIH69 small cell lung carcinoma cells. Oncology 67: 310-319, 2004.

24. Shin BA, Yoo HG, Kim HS, Kim MH, Hwang YS, Chay KO, Lee KY, Ahn BW and Jung YD: P38 MAPK pathway is involved in the urokinase plasminogen activator expression in human gastric SNU-638 cells. Oncol Rep 10: 1467-1471, 2003.

25. Kunapuli P, Kasyapa CS, Hawthorn L and Cowell JK: LGI1, a putative tumor metastasis suppressor gene, controls in vitro invasiveness and expression of matrix metalloproteinases in glioma cells through the ERK1/2 pathway. J Biol Chem 279: 23151-23157, 2004.

26. Andersson U, Guo D, Malmer B, Bergenheim AT, Brannstrom T, Hedman $\mathrm{H}$ and Henriksson R: Epidermal growth factor receptor family (EGFR, ErbB2-4) in gliomas and meningiomas. Acta Neuropathol (Berl) 108: 135-142, 2004.

27. Carroll RS, Black PM, Zhang J, Kirsch M, Percec I, Lau N and Guha A: Expression and activation of epidermal growth factor receptors in meningiomas. J Neurosurg 87: 315-323, 1997.

28. Wild-Bode C, Weller M, Rimner A, Dichgans J and Wick W: Sublethal irradiation promotes migration and invasiveness of glioma cells: implications for radiotherapy of human glioblastoma. Cancer Res 61: 2744-2750, 2001.

29. Lakka SS, Gondi CS and Rao JS: Proteases and glioma angiogenesis. Brain Pathol 15: 327-341, 2005.

30. Akimoto T, Hunter NR, Buchmiller L, Mason K, Ang KK and Milas L: Inverse relationship between epidermal growth factor receptor expression and radiocurability of murine carcinomas. Clin Cancer Res 5: 2884-2890, 1999.

31. Kjoller L: The urokinase plasminogen activator receptor in the regulation of the actin cytoskeleton and cell motility. Biol Chem 383: 5-19, 2002.

32. Blasi $F$ and Carmeliet P: uPAR: a versatile signalling orchestrator. Nat Rev Mol Cell Biol 3: 932-943, 2002.

33. Resnati M, Pallavicini I, Wang JM, Oppenheim J, Serhan CN, Romano $\mathrm{M}$ and Blasi F: The fibrinolytic receptor for urokinase activates the G protein-coupled chemotactic receptor FPRL1/ LXA4R. Proc Natl Acad Sci USA 99: 1359-1364, 2002. 
34. Mandriota SJ, Seghezzi G, Vassalli JD, Ferrara N, Wasi S, Mazzieri R, Mignatti P and Pepper MS: Vascular endothelial growth factor increases urokinase receptor expression in vascular endothelial cells. J Biol Chem 270: 9709-9716, 1995.

35. Pepper MS, Matsumoto K, Nakamura T, Orci L and Montesano R: Hepatocyte growth factor increases urokinase-type plasminogen activator (u-PA) and u-PA receptor expression in Madin-Darby canine kidney epithelial cells. J Biol Chem 267: 20493-20496, 1992.

36. Sliva D: Signaling pathways responsible for cancer cell invasion as targets for cancer therapy. Curr Cancer Drug Targets 4: 327-336, 2004.

37. Dano K, Behrendt N, Hoyer-Hansen G, Johnsen M, Lund LR, Ploug $\mathrm{M}$ and Romer J: Plasminogen activation and cancer. Thromb Haemost 93: 676-681, 2005.

38. Gondi CS, Kandhukuri N, Dinh DH, Gujrati M and Rao JS: Down-regulation of UPAR and uPA activates caspase-mediated apoptosis and inhibits the PI3K/AKT pathway. Int J Oncol 31: 19-27, 2007.

39. Lakka SS, Rajagopal R, Rajan MK, Mohan PM, Adachi Y, Dinh DH, Olivero WC, Gujrati M, Ali-Osman F, Roth JA, Yung WK, Kyritsis AP and Rao JS: Adenovirus-mediated antisense urokinase-type plasminogen activator receptor gene transfer reduces tumor cell invasion and metastasis in nonsmall cell lung cancer cell lines. Clin Cancer Res 7: 1087-1093, 2001.

40. Mohanam S, Jasti SL, Kondraganti SR, Chandrasekar N, Kin Y, Fuller GN, Lakka SS, Kyritsis AP, Dinh DH, Olivero WC, Gujrati M, Yung WK and Rao JS: Stable transfection of urokinase-type plasminogen activator antisense construct modulates invasion of human glioblastoma cells. Clin Cancer Res 7: 2519-2526, 2001
41. Subramanian R, Gondi CS, Lakka SS, Jutla A and Rao JS: siRNA-mediated simultaneous downregulation of uPA and its receptor inhibits angiogenesis and invasiveness triggering apoptosis in breast cancer cells. Int J Oncol 28: 831-839, 2006.

42. De Bock CE and Wang Y: Clinical significance of urokinasetype plasminogen activator receptor (UPAR) expression in cancer. Med Res Rev 24: 13-39, 2004.

43. Kuhn W, Pache L, Schmalfeldt B, Dettmar P, Schmitt M, Janicke $\mathrm{F}$ and Graeff $\mathrm{H}$ : Urokinase (uPA) and PAI-1 predict survival in advanced ovarian cancer patients (FIGO III) after radical surgery and platinum-based chemotherapy. Gynecol Oncol 55: 401-409, 1994.

44. D'Alessio S, Margheri F, Pucci M, Del Rosso A, Monia BP, Bologna M, Leonetti C, Scarsella M, Zupi G, Fibbi G and Del RM: Antisense oligodeoxynucleotides for urokinaseplasminogen activator receptor have anti-invasive and antiproliferative effects in vitro and inhibit spontaneous metastases of human melanoma in mice. Int J Cancer 110: 125-133, 2004.

45. Speake WJ, Dean RA, Kumar A, Morris TM, Scholefield JH and Watson SA: Radiation induced MMP expression from rectal cancer is short lived but contributes to in vitro invasion. Eur J Surg Oncol 31: 869-874, 2005. 